\title{
Cell adhesion molecules and exercise
}

Yunsuk Koh'

Jinkyung Park ${ }^{2}$

'Department of Health, Human Performance, and Recreation, Baylor University, Waco, TX, USA; ${ }^{2}$ Department of Kinesiology, George Mason University, Manassas, VA, USA

Correspondence: Yunsuk Koh Department of Health, Human Performance, and Recreation, Baylor University, One Bear Place \#973 I3,

Waco, TX 76798-7313, USA

$\mathrm{Tel}+\mathrm{I} 2547104002$

Fax + I 2547103527

Email yunsuk_koh@baylor.edu
This article was published in the following Dove Press journal:

Journal of Inflammation Research

Background: An extensive systematic review was undertaken in the current literature in order to explore the role of different types and intensities of exercise in cell adhesion molecules (CAMs), one of the markers of vascular inflammation, endothelial dysfunction, and atherosclerosis.

Methods: Twenty-eight qualifying studies were extensively reviewed to examine the effects of different intensities (low-to-moderate vs. high) and types (aerobic vs. resistance) of exercise on intercellular adhesion molecule-1, vascular cell adhesion molecule-1, and selectins.

Results: Low-to-moderate intensity aerobic exercise favorably decreased CAMs in a variety of subject populations, while CAMs momentarily increased immediately following high-intensity aerobic exercise, which then returned to the pre-exercise level within several hours post-exercise. Resistance exercise, regardless of its intensity, did not significantly influence CAMs.

Conclusion: It is evident that the responses of CAMs are dependent upon the type and intensity of exercise performed. The most common, favorable outcome was a decrease in CAMs following low-to-moderate intensity aerobic exercise, while high-intensity aerobic exercise showed a short-lived, momentary increase in CAMs, which returned to the pre-exercise level within a few hours post-exercise. Resistance exercise, regardless of its intensity, neither significantly increased nor decreased CAMs. Future studies should focus more on the role of exercise in both soluble and membrane-bound CAMs as well as proinflammatory cytokines related to atherosclerosis in order to develop specific exercise programing that can effectively improve vascular inflammation and endothelial health.

Keywords: ICAM-1, VCAM-1, endothelial dysfunction, inflammation, cardiovascular diseases, atherosclerosis

\section{Introduction}

Atherosclerosis is the most common type of coronary heart disease, which is the leading cause of mortality attributable to cardiovascular disease (CVD). ${ }^{1}$ Emerging evidence shows that chronic inflammation may play a critical role in the development of atherosclerosis and suggests that understanding the role of specific inflammatory proteins and pathways associated with atherosclerosis can provide more effective treatments and guidelines for lowering the risk of cardiovascular events. One of the strong biomarkers for vascular inflammation is cell adhesion molecules (CAMs), which are also closely related to a variety of proinflammatory cytokines such as IL-1, IL-8, and tumor necrosis factor alpha. ${ }^{2}$

CAMs are glycoproteins expressed on the surface of various cells and play a crucial role in many cellular processes, including binding with the extracellular matrix. ${ }^{3}$ Elevated expression and activity of CAMs are indicative of inflammation, endothelial dysfunction, and atherosclerosis. ${ }^{4}$ Both intracellular adhesion molecule-1 (ICAM-1) and vascular cell 
adhesion molecule-1 (VCAM-1) are the transmembrane glycoproteins that are responsible for transmigrating leukocytes into the vascular intima. The four major steps of leukocyte adhesion cascade by CAMs involve 1) capturing, 2) rolling, 3) adhesion, and 4) transmigration (Figure 1). Selectins are involved in the first two steps of the adhesion cascade. The interaction between P- and E-selectins allows the circulating leukocytes to adhere to the endothelial wall (capturing) and then to move along the endothelium (rolling). Although L-selectin is known to participate in the secondary capturing process, the exact role of L-selectin in inflammation and atherosclerosis is not well known. The next step is "adhesion" of the leukocyte integrins to ICAM-1 and VCAM-1, which further transmigrate leukocytes into the damaged vascular intima, leading to the formation of the atherosclerotic lesion. ${ }^{5}$

Thus, elevated CAMs promote vascular inflammation ${ }^{3,5}$ and accumulation of macrophages within the intima, ${ }^{6}$ which consequently accelerate the atherosclerotic progression. Directly measuring the actual endothelial expression and activity of CAMs on human tissues is quite challengeable, while soluble form of CAMs (sCAMs) in the circulation can be conveniently obtained. These sCAMs indirectly reflect the rate of endothelial expression and activation of CAMs since they are shed from the surface of endothelial and lymphocyte cells after being activated. ${ }^{7}$ Therefore, sCAMs are considered the strong biomarkers of inflammation, endothelial dysfunction, and atherosclerosis. ${ }^{8}$

Exercise has been widely recommended as one of the non-pharmacological approaches to improve and prevent inflammation and CVD through various physiological and biochemical mechanisms. Although some existing evidence suggests that CAMs may change to a certain extent in response to exercise, the results are equivocal. Moreover, there is no clear understanding regarding the responses of CAMs to certain types and intensities of exercise. ${ }^{9-16}$ Understanding the role of different types and intensities of exercise in CAMs will not only enhance our current knowledge about this research topic but also provide insights into the development of specific exercise programming in order to effectively improve vascular inflammation and endothelial health. Therefore, the purpose of the present study was to examine the current literature exploring the responses of CAMs, including ICAM-1, VCAM-1, E-selectins, P-selectins, and L-selectins, following different types (aerobic vs. resistance) and intensities (low-to-moderate vs. high) of exercise in a variety of subject populations.

\section{Methods}

The literature search was conducted using a Web of Science database for the journal articles written in English and published between 1997 and 2017. The initial search was conducted using the terms "exercise" and "cell adhesion molecules". From these pre-selected articles, we further searched any relevant articles cited in each article. In the current review, however, we only included the experimental-design original research, containing the following terms: "exercise", "ICAM-1", "VCAM-1", "sICAM-1", "sVCAM-1", "selectins", "E-selectin", "P-selectin", or "L-selectin" in humans (Figure 2). Both men and women of any ages with or without

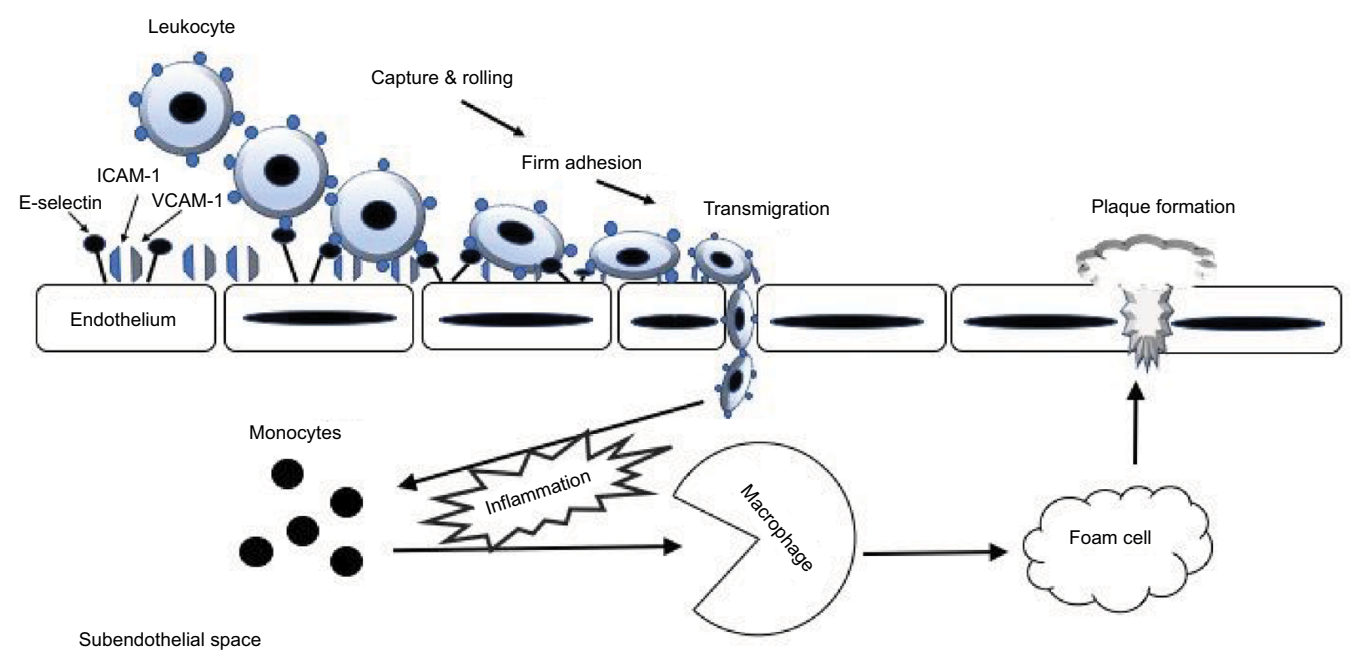

Figure I The role of CAMs in the leukocyte adhesion cascade during endothelial inflammation. Abbreviation: CAMs, cell adhesion molecules. 

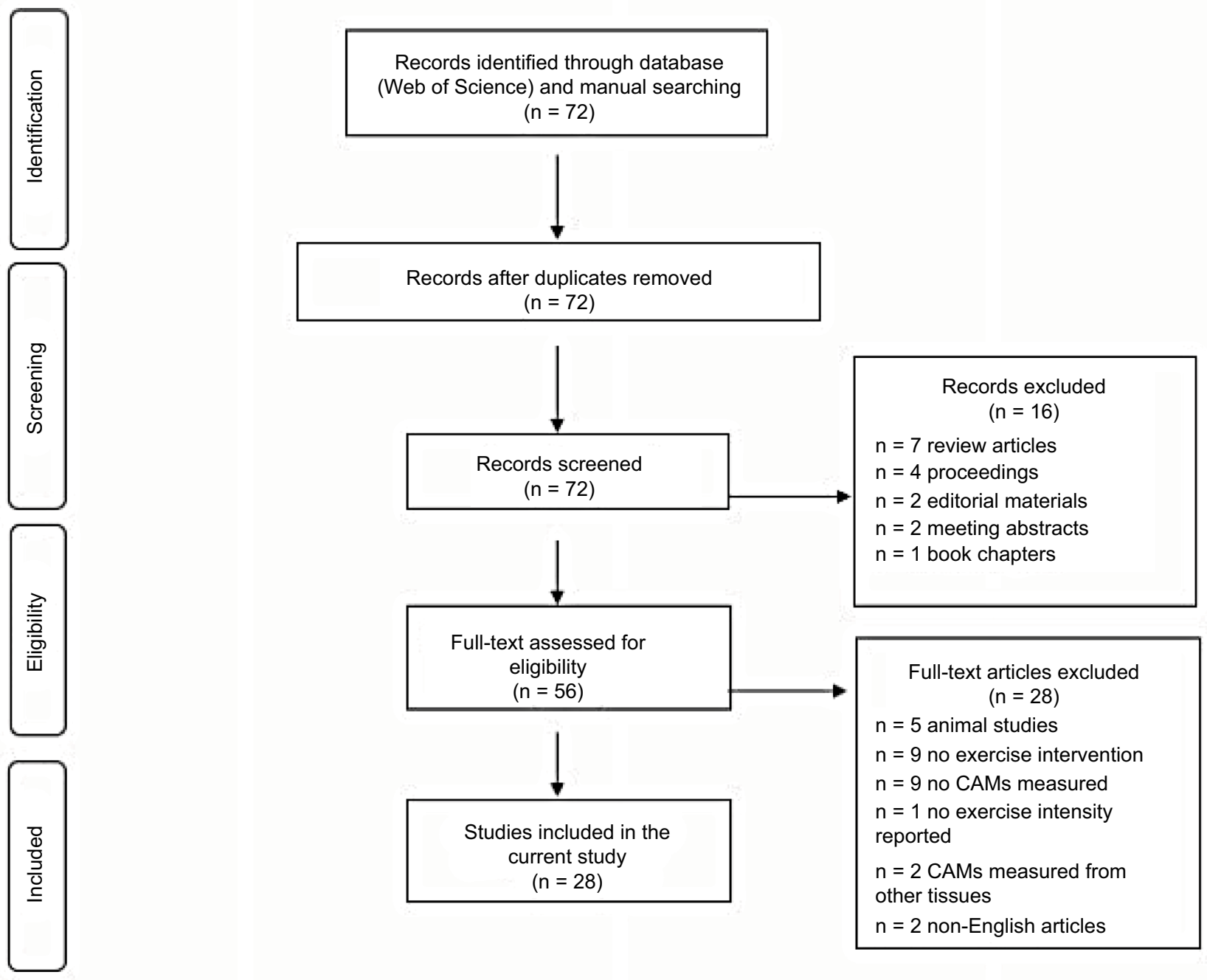

Figure 2 A flow diagram of literature search and selection.

Abbreviation: CAMs, cell adhesion molecules.

any known cardiometabolic diseases were included. As for the exercise intervention, any intensities (low-to-moderate and high), types (aerobic and resistance), and duration (acute and chronic) of exercise were considered.

\section{Results}

As shown in Figure 2, the initial search using the terms "exercise" and "cell adhesion molecule" through a Web of Science database found a total of 72 journal articles. A total of 16 articles were excluded due to the following reasons: review articles $(n=7)$, conference proceedings $(n=4)$, editorial materials $(n=2)$, meeting abstracts $(n=2)$, and book chapter $(n=1)$. A total of 56 full-text articles were further examined for eligibility, and 28 articles were excluded due to animal subjects $(n=5)$, no exercise intervention $(n=9)$, no CAMs measured $(n=9)$, exercise intensity not reported $(\mathrm{n}=1)$, CAMs measured in tissues other than plasma or lymphocytes $(n=2)$, and non-English articles $(n=2)$. As a result, a total of 28 qualifying articles were finally selected and included in the current review (Table 1).

Twenty-one of 28 studies employed an aerobic exercise intervention (mixed intensity $=3$, moderate intensity $=13$, and high intensity $=7$ ), while only 5 studies used resistance exercise as an exercise intervention (low intensity $=1$, moderate intensity $=3$, and high intensity $=1$ ). A majority of the studies in the current review reported either no change or decrease in CAMs following low-to-moderate intensity aerobic exercise, whereas high-intensity aerobic exercise increased CAMs. Regardless of intensity, resistance exercise showed no significant changes in CAMs.

\section{Discussion}

\section{Low-to-moderate intensity exercise}

Aerobic exercise

A majority of the studies that we reviewed reported either no change $e^{16,17,24}$ or a decrease $e^{9,14,15,19,22,23,26,27}$ in both soluble 


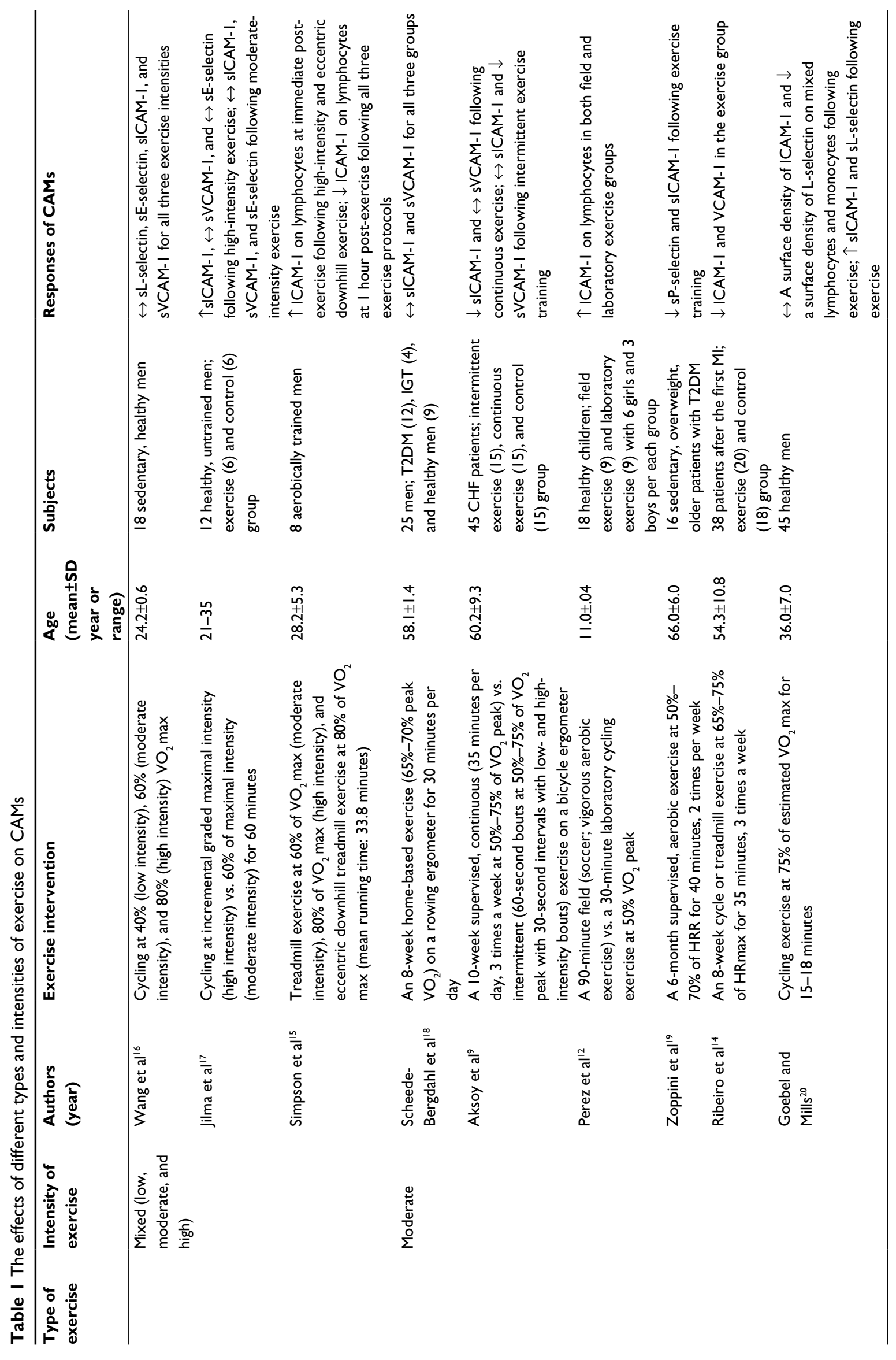




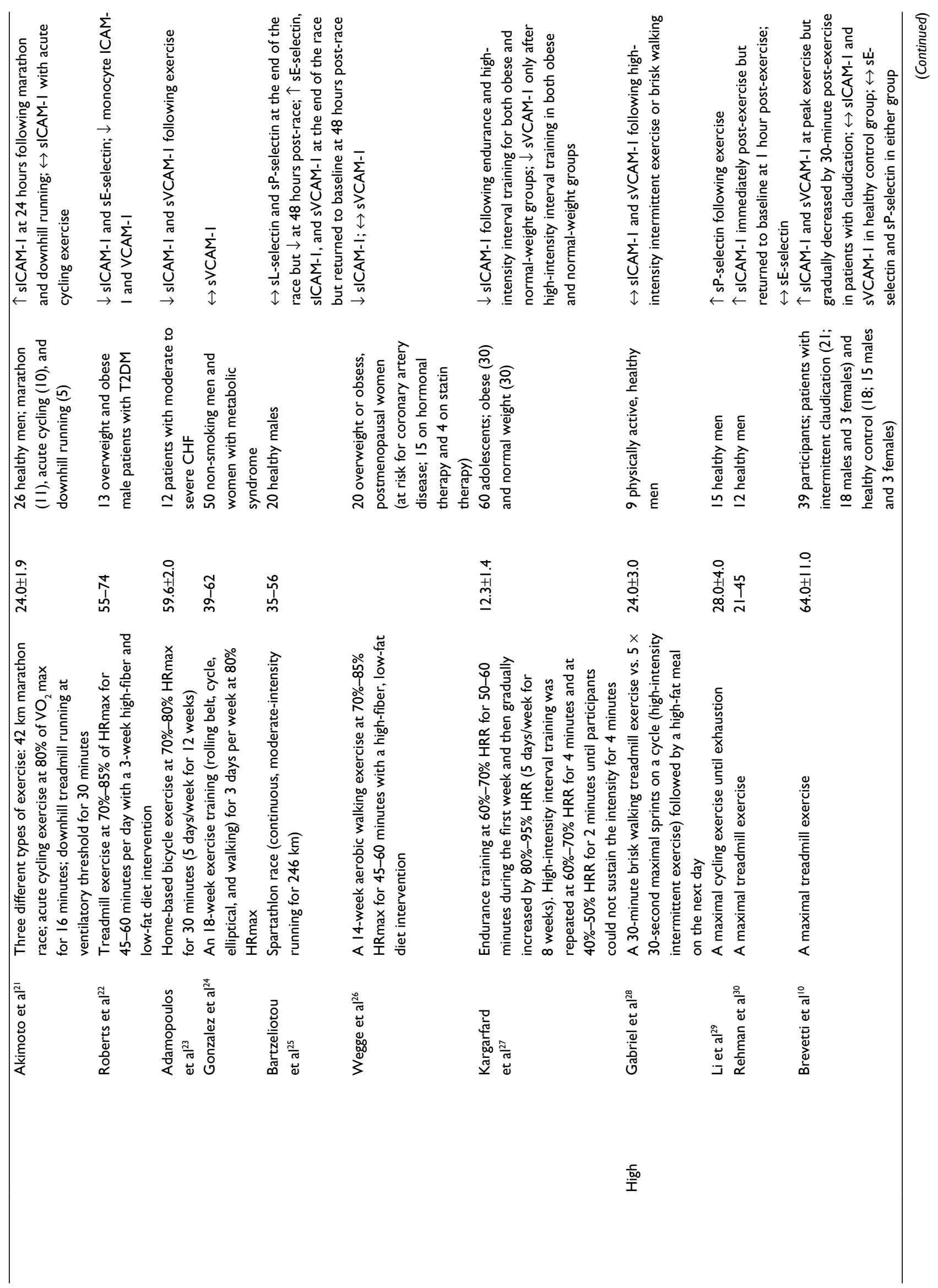




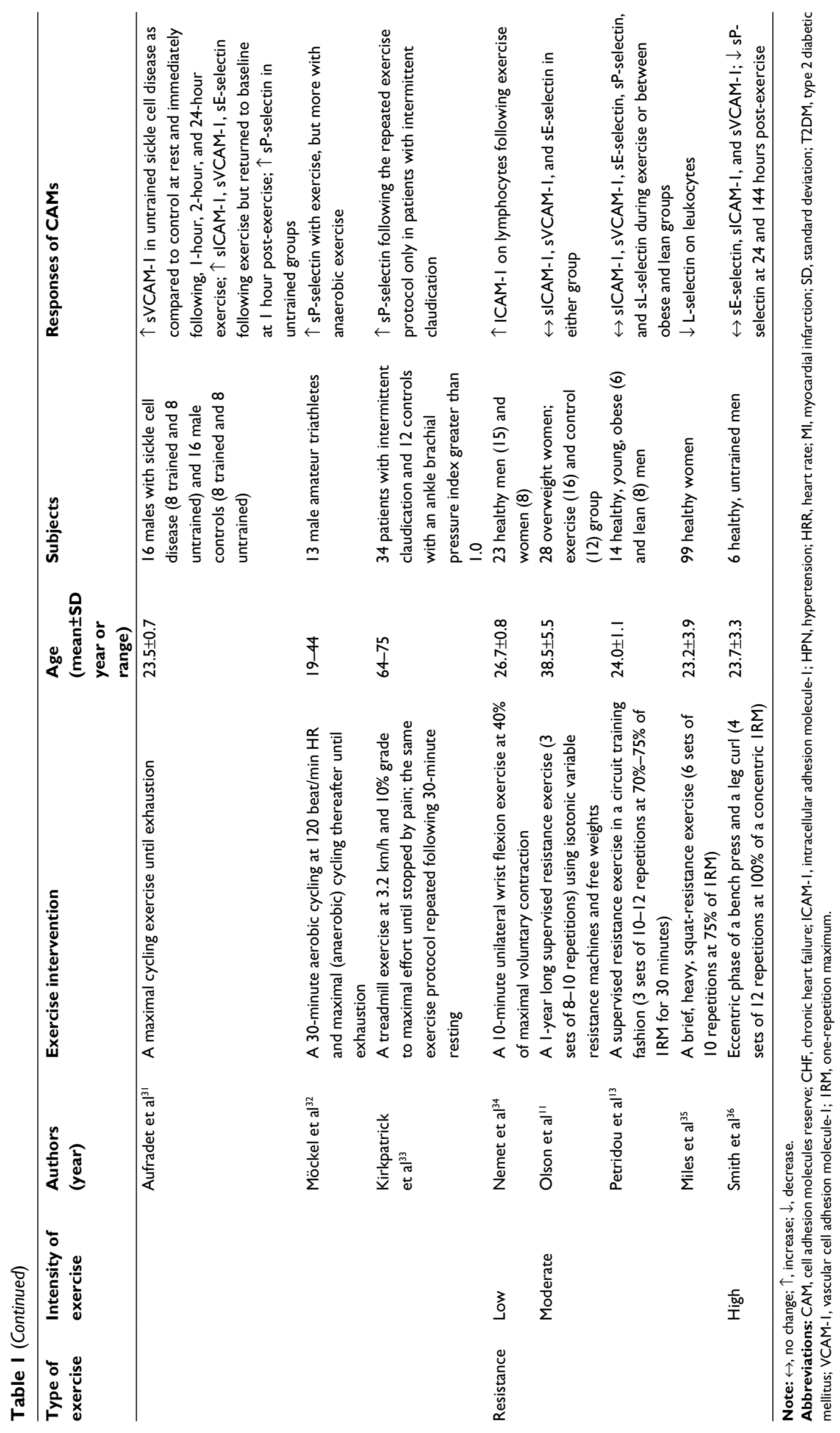


and lymphocyte CAMs following low-to-moderate intensity aerobic exercise in a variety of subject populations. For instance, both obese and normal-weight adolescents who participated in an 8-week moderate-intensity endurance or interval training showed significantly decreased sICAM-1 and sVCAM-1. When compared with endurance training, interval training showed more effective results in reducing these molecules. ${ }^{27}$ Individuals with a certain type of cardiometabolic diseases, such as chronic heart failure (CHF), type 2 diabetic mellitus (T2DM), or myocardial infarction, had reduced sICAM-1, sVCAM-1, and sP-selectin following moderate-intensity exercise training for 8-12 weeks. ${ }^{9,14,19,23}$ Overweight and obese postmenopausal women who were on a hormonal therapy had decreased sICAM-1 following a 2-week moderate-intensity walking exercise and a high-fiber, low-fat diet intervention. ${ }^{26}$ Similarly, Roberts et al also observed a decrease in SICAM-1, sE-selectin, and monocyte ICAM and VCAM following moderate-intensity exercise accompanied by a high-fiber, low-fat diet in overweight and obese patients with T2DM. ${ }^{22}$ A significant correlation was reported between the improvement in $\mathrm{VO}_{2}$ max and the reduction in sICAM-1 and sVCAM-1 in patients with $\mathrm{CHF}$, suggesting that improved physical fitness may positively influence endothelial health. ${ }^{23}$ Not only untrained individuals but also trained individuals had significantly lowered expression of lymphocyte ICAM-1 following an acute moderate-intensity treadmill exercise. However, this exercise-induced decrease in lymphocyte ICAM-1 returned to the pre-exercise level at 24 hours post-exercise, suggesting that an acute aerobic exercise may have a short-lived effect on lymphocyte ICAM-1 in trained individuals who had normal levels of this molecule. ${ }^{15}$

Several studies have reported no changes in sICAM-1, sVCAM-1, or sE-selectin following low-to-moderate intensity aerobic exercise training. ${ }^{15-18,24}$ Both sedentary and physically active men ${ }^{15-17}$ did not have any change in SICAM-1, sVCAM-1, sE-selectin, and lymphocyte ICAM-1 following exercise training $\left(40 \%-60 \% \mathrm{VO}_{2} \max \right)$. In addition, patients with either T2DM or impaired glucose tolerance and their healthy counterparts did not have any change in sICAM-1 or sVCAM-1 following an 8-week home-based exercise training. ${ }^{18}$ sVCAM-1 in middle-aged men and women with metabolic syndrome was not altered after an 18-week moderate intensity aerobic exercise training. ${ }^{24}$ By contrast, a few studies reported an increase in either soluble ${ }^{12,25,31}$ or lymphocyte ICAM-1 ${ }^{21}$ following an acute bout of maximal or moderate-intensity exercise. Young males with or without sickle cell disease, regardless of training status, had increased sICAM-1, sVCAM-1, and sE-selectin following a maximal cycling exercise, which in turn returned to the baseline value at 1 hour post-exercise. ${ }^{31}$ After completing an ultra-distance race of continuous, moderate-intensity running $(246 \mathrm{~km})$, male runners had an increase in sE-selectin, sVCAM-1, and sICAM-1 at the end of the race; however, these elevated CAMs returned to the pre-race values at 48 hours post-race. Unlike chronic aerobic exercise training, an acute bout of maximal or moderate intensity with prolonged duration of aerobic exercise may transiently raise sCAMs. However, it should be noted that elevated sCAMs, particularly sEselectin and SICAM-1, in the circulation are not necessarily indicative of elevated endothelial inflammation or dysfunction, since it has been proposed that elevated soluble CAMs may exert some protective effects on the endothelium. For instance, elevated sE-selectin in the circulation may inhibit the adhesion and binding process of circulating leukocytes to the endothelium by competing with its ligand on circulating leukocytes. Thus, this process will eventually prevent these circulating leukocytes from recognizing and binding to their corresponding membrane-bound form of E-selectin. ${ }^{37,38}$

Interestingly, sL-selectin and $\mathrm{sP}$-selectin were significantly reduced at 48 hours post-race, and the study suggested that an exercise-induced decrease in sL-selectin and $\mathrm{sP}$-selectin may be due to the restoration process of the endothelial barrier and vascular function. ${ }^{25}$ Similarly, sICAM-1 in healthy men was elevated at 24 hours after 42 $\mathrm{km}$ marathon running or downhill running performed at the ventilatory threshold for 30 minutes, whereas SICAM-1 did not change following moderate-intensity cycling exercise. ${ }^{21}$ In fact, one of the possible explanations in which sICAM-1 increased following marathon or downhill running, but not cycling exercise, despite all of the three exercises performed at moderate intensity, may be due to a severe muscle damage possibly resulted from a prolonged, strenuous exercise. Another study examining the effect of a moderate-intensity laboratory cycling exercise $\left(50 \% \mathrm{VO}_{2}\right.$ peak for 30 minutes) versus a vigorous field exercise (soccer for 90 minutes) on lymphocyte ICAM-1 in healthy children reported an increase in ICAM-1 following both exercise protocols. ${ }^{12}$ However, it should be noted that two exercise groups in this study had significantly different age, body mass, and height at baseline, which may affect the response of CAMs to exercise, since the development of children's immune system is dependent on their physical maturity and may affect immune responses differently following exercise. 


\section{Resistance exercise}

Only a few studies have previously examined the role of lowto-moderate intensity resistance exercise in CAMs. ${ }^{11,13,34,35}$ Thus, the information presented in the current study is limited and based only on the findings of these studies. Overall, it seems that low-to-moderate intensity resistance exercise may not significantly influence CAMs. Both healthy lean and obese men who performed a single bout of moderateintensity resistance exercise at $70 \%-75 \%$ of one repetition maximum ${ }^{13}$ and overweight women who participated in a year-long low-to-moderate-intensity resistance exercise program (8-10 RM for 3 sets $)^{11}$ did not significantly change sICAM-1, sVCAM-1, or sE-selectin. However, leukocyte L-selectin in healthy women decreased following moderateintensity squat exercise. ${ }^{35} \mathrm{By}$ contrast, one study reported an increase in the expression of lymphocyte ICAM-1 following low-intensity wrist flexion exercise in healthy men and women, and the study suggested that exercise on a specific local body part may influence overall vascular immune response. ${ }^{34}$ However, it should be noted that this particular study is not entirely comparable to the other studies in our review examining the effect of different types and intensities of exercise on CAMs since there is no strong evidence suggesting that the local effect of exercise can actually lead to a systemic response of CAMs.

Thus, based on the studies we reviewed, changes in CAMs in response to low-to-moderate intensity exercise seems to be dependent upon the type of exercise performed. Low-tomoderate intensity aerobic exercise can improve endothelial inflammation by downregulating the expression of lymphocyte CAMs and lowering sCAMs in the circulation, while resistance exercise at low-to-moderate intensity may not significantly alter CAMs. Not only healthy individuals but also patients with a certain form of cardiometabolic diseases or individuals with the high risk of CVD benefit from lowto-moderate intensity exercise by lowering both soluble and membrane-bound CAMs. Given the fact that each soluble or membrane-bound form of CAMs may independently play a significant role in the endothelial adhesion process, examining the responses and mechanisms of both forms of CAMs will enhance our current knowledge about the roles of CAMs and the effects of exercise on the endothelial health.

\section{High-intensity exercise}

\section{Aerobic exercise}

A few studies have reported no change in sCAMs following high-intensity aerobic exercise in sedentary or active men, ${ }^{16,28}$ whereas a majority of the studies have reported an acute increase in sCAMs immediately following highintensity exercise..$^{10,15,17,30,31}$ However, most studies showed that this exercise-induced increase in sCAMs returned to the pre-exercise values within several hours post-exercise. For instance, Jilma et al reported an elevation of SICAM-1, but not sVCAM-1 or sE-selectin, following high-intensity cycling exercise in untrained men. ${ }^{17}$ Similarly, healthy men had an increase in sICAM-1 immediately following a maximal treadmill exercise, which then returned to the baseline values at 1 hour post-exercise, whereas $\mathrm{SE}$-selectin did not change. The authors suggested that an increase in sICAM-1 may be due to the direct stimulation of adrenoceptor on lymphocytes or endothelial cells. ${ }^{30}$ Simpson et al also observed a significant increase in lymphocyte ICAM-1 immediately following either a high-intensity treadmill exercise or an eccentric downhill exercise in aerobically trained men; however, this exercise-induced increase was no longer significant after 1 hour post-exercise. ${ }^{15}$ Several studies reported an increase in soluble or membrane-bound P-selectin following maximal exercise in healthy individuals and patients with sickle cell disease or intermittent claudication, ${ }^{29,31,32,33}$ indicating that strenuous exercise may lead to an activation of platelets. ${ }^{32}$ Kirkpatrick et al suggested that a progressive activation of sP-selectin induced by high-intensity exercise may negatively impact cardiovascular health. ${ }^{33}$ Individuals with sickle cell disease also had increased sICAM-1, sVCAM-1, and sEselectin following a maximal exercise testing, regardless of training status. It was also noted that untrained individuals with sickle cell disease had significantly higher levels of sCAMs than the trained counterparts at rest and postexercise, ${ }^{31}$ suggesting that improved physical fitness may attenuate the exercise-induced elevation in SCAMs. Although patients with claudication had an increase in both sICAM-1 and SVCAM-1 at peak exercise, they gradually decreased by 30 minutes post-exercise, whereas the healthy counterparts did not have any change in these molecules. The authors of the aforementioned study speculated that an exerciseinduced increase in SICAM- 1 and SVCAM- 1 could reflect inflammatory activity rather than endothelial activation. ${ }^{10}$ However, several other studies have rather suggested that an exercise-induced increase in sCAMs may be mainly caused by the shedding of these adhesion molecules due to elevated shear stress particularly during high-intensity exercise ${ }^{35,36,39}$ or possibly by an exercise-induced hemoconcentration. ${ }^{40}$

\section{Resistance exercise}

In our search, there was only one previous study examining the effect of high-intensity resistance exercise on CAMs in 
healthy untrained men and reported no changes in SICAM-1, sVCAM-1, or sE-Selectin, whereas lymphocyte P-selectin was reduced at 24 and 144 hours post-exercise. ${ }^{36}$

Based on the studies we reviewed, the responses of CAMs to high-intensity exercise is dependent upon the type of exercise performed. High-intensity aerobic exercise may acutely increase sICAM-1 following exercise, while high-intensity resistance exercise may not significantly change CAMs. As mentioned earlier, it is possible that muscle damage induced by high-intensity exercise may affect the responses of postexercise CAMs. ${ }^{21}$ Therefore, high-intensity exercise, particularly prolonged, strenuous aerobic exercise, may result in an acute increase in CAMs during and shortly after exercise. However, this acute change was relatively short-lived and not significant any longer after several hours following exercise.

\section{Conclusion}

Exercise is clearly an immune stimulator and considered one of the potential underlying mechanisms for the CAM metabolism. According to the current literature, CAMs can be affected by the type and intensity of exercise performed. The most common, favorable outcome observed in both health individuals and patients with cardiometabolic diseases was a decrease in CAMs following low-to-moderate intensity aerobic exercise, while high-intensity aerobic exercise momentarily elevated these molecules immediately following exercise. However, it was noted that this exercise-induced increase in CAMs returned to the pre-exercise level and may be caused by the shedding of these molecules due to increased shear stress. There was a major paucity of research exploring the role of resistance exercise in CAMs; thus, it was difficult to conclude the exact role of resistance exercise in CAMs due to a lack of knowledge and information. Nevertheless, the current literature showed that resistance exercise, regardless of its intensity, neither significantly increased nor decreased CAMs. Some of the limitations for the current review include a subjective exercise intensity used in the previous studies and the various methods of quantitative measurement of CAMs using different forms of biological samples. Additionally, it is currently not clearly understood how sCAM is related to its membrane-bound variants. Thus, it is highly recommended for future studies to further explore the effects of exercise on both soluble and membrane-bound CAMs as well as proinflammatory cytokines related to atherosclerosis in order to provide insights into the development of exercise programing that can effectively improve vascular inflammation and endothelial health. Additionally, it is imperative to examine the role of resistance exercise in CAMs and the relationship between CAMs and inflammatory markers particularly during prolonged, strenuous aerobic exercise at high intensity in a variety of subject populations.

\section{Disclosure}

The authors report no conflicts of interest in this work.

\section{References}

1. Benjamin EJ, Virani SS, Callaway CW, et al; American Heart Association Council on Epidemiology and Prevention Statistics Committee and Stroke Statistics Subcommittee. Heart disease and stroke statistics-2018 update: a report from the American Heart Association. Circulation. 2018;137(12):e67-e492.

2. Golia E, Limongelli G, Natale F, et al. Inflammation and cardiovascular disease: from pathogenesis to therapeutic target. Curr Atheroscler Rep. 2014;16(9):435.

3. Galkina E, Ley K. Vascular adhesion molecules in atherosclerosis. Arterioscler Thromb Vasc Biol. 2007;27(11):2292-2301.

4. Libby P. Inflammation in atherosclerosis. Nature. 2002;420(6917): 868-874.

5. Huo Y, Ley K. Adhesion molecules and atherogenesis. Acta Physiol Scand. 2001;173(1):35-43.

6. Hansson GK, Hermansson A. The immune system in atherosclerosis. Nat Immunol. 2011;12(3):204-212.

7. Abe Y, El-Masri B, Kimball KT, et al. Soluble cell adhesion molecules in hypertriglyceridemia and potential significance on monocyte adhesion. Arterioscler Thromb Vasc Biol. 1998;18(5):723-731.

8. Pigott R, Dillon LP, Hemingway IH, Gearing AJ. Soluble forms of E-selectin, ICAM-1 and VCAM-1 are present in the supernatants of cytokine activated cultured endothelial cells. Biochem Biophys Res Commun. 1992;187(2):584-589.

9. Aksoy S, Findikoglu G, Ardic F, Rota S, Dursunoglu D. Effect of 10 -week supervised moderate-intensity intermittent vs. continuous aerobic exercise programs on vascular adhesion molecules in patients with heart failure. Am J Phys Med Rehabil. 2015;94(10 Suppl 1): 898-911.

10. Brevetti G, De Caterina M, Martone VD, et al. Exercise increases soluble adhesion molecules ICAM-1 and VCAM-1 in patients with intermittent claudication. Clin Hemorheol Microcirc. 2001;24(3):193-199.

11. Olson TP, Dengel DR, Leon AS, Schmitz KH. Changes in inflammatory biomarkers following one-year of moderate resistance training in overweight women. Int J Obes (Lond). 2007;31(6):996-1003.

12. Perez CJ, Nemet D, Mills PJ, Scheet TP, Ziegler MG, Cooper DM. Effects of laboratory versus field exercise on leukocyte subsets and cell adhesion molecule expression in children. Eur J Appl Physiol. 2001;86(1):34-39.

13. Petridou A, Chatzinikolaou A, Fatouros I, et al. Resistance exercise does not affect the serum concentrations of cell adhesion molecules. Br J Sports Med. 2007;41(2):76-79; discussion 79.

14. Ribeiro F, Alves AJ, Teixeira M, et al. Exercise training increases interleukin-10 after an acute myocardial infarction: a randomised clinical trial. Int J Sports Med. 2012;33(3):192-198.

15. Simpson RJ, Florida-James GD, Whyte GP, Guy K. The effects of intensive, moderate and downhill treadmill running on human blood lymphocytes expressing the adhesion/activation molecules CD54 (ICAM-1), CD18 (beta2 integrin) and CD53. Eur J Appl Physiol. 2006;97(1):109-121.

16. Wang JS, Chen YW, Chow SE, Ou HC, Sheu WH. Exercise paradoxically modulates oxidized low density lipoprotein-induced adhesion molecules expression and trans-endothelial migration of monocyte in men. Thromb Haemost. 2005;94(4):846-852.

17. Jilma B, Eichler HG, Stohlawetz P, et al. Effects of exercise on circulating vascular adhesion molecules in healthy men. Immunobiology. 1997;197(5):505-512. 
18. Scheede-Bergdahl C, Benee Olsen D, Reving D, Boushel R, Dela F. Cardiovascular disease markers in type 2 diabetes: the effects of a moderate home-based exercise training programme. Diab Vasc Dis Res. 2009;6(4):291-296.

19. Zoppini G, Targher G, Zamboni C, et al. Effects of moderate-intensity exercise training on plasma biomarkers of inflammation and endothelial dysfunction in older patients with type 2 diabetes. Nutr Metab Cardiovasc Dis. 2006;16(8):543-549.

20. Goebel MU, Mills PJ. Acute psychological stress and exercise and changes in peripheral leukocyte adhesion molecule expression and density. Psychosom Med. 2000;62(5):664-670.

21. Akimoto T, Furudate M, Saitoh M, et al. Increased plasma concentrations of intercellular adhesion molecule-1 after strenuous exercise associated with muscle damage. Eur J Appl Physiol. 2002;86(3):185-190.

22. Roberts CK, Won D, Pruthi S, Lin SS, Barnard RJ. Effect of a diet and exercise intervention on oxidative stress, inflammation and monocyte adhesion in diabetic men. Diabetes Res Clin Pract. 2006;73(3):249-259.

23. Adamopoulos S, Parissis J, Kroupis C, et al. Physical training reduces peripheral markers of inflammation in patients with chronic heart failure. Eur Heart J. 2001;22(9):791-797.

24. Gonzalez IP, Madariaga EL, Aviles CR, et al. Eighteen-week exercise and nutritional education program did not modify the serum levels of sVCAM-1 and sCD40-L in subjects with metabolic syndrome. Labmedicine. 2010;41(4):231-234.

25. Bartzeliotou AI, Margeli AP, Tsironi M, et al. Circulating levels of adhesion molecules and markers of endothelial activation in acute inflammation induced by prolonged brisk exercise. Clin Biochem . 2007;40(11):765-770.

26. Wegge JK, Roberts CK, Ngo TH, Barnard RJ. Effect of diet and exercise intervention on inflammatory and adhesion molecules in postmenopausal women on hormone replacement therapy and at risk for coronary artery disease. Metabolism. 2004;53(3):377-381.

27. Kargarfard M, Lam ETC, Shariat A, et al. Effects of endurance and high intensity training on ICAM-1 and VCAM-1 levels and arterial pressure in obese and normal weight adolescents. Phys Sportsmed. 2016;44(3):208-216.

28. Gabriel B, Ratkevicius A, Gray P, Frenneaux MP, Gray SR. Highintensity exercise attenuates postprandial lipaemia and markers of oxidative stress. Clin Sci (Lond). 2012;123(5):313-321.
29. Li N, Wallén NH, Hjemdahl P. Evidence for prothrombotic effects of exercise and limited protection by aspirin. Circulation. 1999;100(13):1374-1379.

30. Rehman J, Mills PJ, Carter SM, Chou J, Thomas J, Maisel AS. Dynamic exercise leads to an increase in circulating ICAM-1: further evidence for adrenergic modulation of cell adhesion. Brain Behav Immun. 1997;11(4): 343-351.

31. Aufradet E, Monchanin G, Oyonno-Engelle S, et al. Habitual physical activity and endothelial activation in sickle cell trait carriers. Med Sci Sports Exerc. 2010;42(11):1987-1994.

32. Möckel M, Ulrich NV, Rocker L, et al. Exhaustive cycle exercise induces P-selectin expression, coagulation, and fibrinolysis activation in ultraendurance athletes. Thromb Res. 1999;94(4):263-269.

33. Kirkpatrick UJ, Mossa M, Blann AD, McCollum CN. Repeated exercise induces release of soluble P-selectin in patients with intermittent claudication. Thromb Haemost. 1997;78(5):1338-1342.

34. Nemet D, Hong S, Mills PJ, Ziegler MG, Hill M, Cooper DM. Systemic vs. local cytokine and leukocyte responses to unilateral wrist flexion exercise. J Appl Physiol (1985). 2002;93(2):546-554.

35. Miles MP, Leach SK, Kraemer WJ, Dohi K, Bush JA, Mastro AM. Leukocyte adhesion molecule expression during intense resistance exercise. J Appl Physiol (1985). 1998;84(5):1604-1609.

36. Smith LL, Anwar A, Fragen M, Rananto C, Johnson R, Holbert D. Cytokines and cell adhesion molecules associated with high-intensity eccentric exercise. Eur J Appl Physiol. 2000;82(1-2):61-67.

37. Blann AD, Tse W, Maxwell SJ, Waite MA. Increased levels of the soluble adhesion molecule E-selectin in essential hypertension. $J$ Hypertens. 1994;12(8):925-928.

38. Vastag M, Skopál J, Voko Z, Csonka E, Nagy Z. Expression of membrane-bound and soluble cell adhesion molecules by human brain microvessel endothelial cells. Microvasc Res. 1999;57(1):52-60.

39. Arber N, Berliner S, Rotenberg Z, et al. Detection of aggregated leukocytes in the circulating pool during stress-demargination is not necessarily a result of decreased leukocyte adhesiveness. Acta Haematol. 1991;86(1):20-24.

40. Tripette J, Connes P, Hedreville M, et al. Patterns of exercise-related inflammatory response in sickle cell trait carriers. Br J Sports Med. 2010;44(4):232-237.
Journal of Inflammation Research

\section{Publish your work in this journal}

The Journal of Inflammation Research is an international, peer-reviewed open access journal that welcomes laboratory and clinical findings on the molecular basis, cell biology and pharmacology of inflammation including original research, reviews, symposium reports, hypothesis formation and commentaries on: acute/chronic inflammation; mediators of

\section{Dovepress}

inflammation; cellular processes; molecular mechanisms; pharmacology and novel anti-inflammatory drugs; clinical conditions involving inflammation. The manuscript management system is completely online and includes a very quick and fair peer-review system. Visit http://www.dove press.com/testimonials.php to read real quotes from published authors. 